\title{
LAS FÁBRICAS DE LA CHARLA EN CHILE: APUNTES PRELIMINARES SOBRE LA MATERIALIDAD Y LA SUBJEIIVIDAD DEL TRABAJO EN LOS CALL CENIERS
}

\section{Areli Escobar Salazar* \\ Universidad Autonóma de Barcelona - España}

Resumen: Este artículo expone los resultados preliminares de la investigación doctoral en Antropología, orientada a conocer la materialidad y la subjetividad del trabajo de los call centers en Chile. Un salario definido por metas inalcanzables, el maltrato, el cansancio de nuevo tipo, la doble extracción del valor productivo y reproductivo de las mujeres trabajadoras y su entorno familiar, la explotación de las capacidades subjetivas, entre otros aspectos, dan cuenta de las nuevas formas que asume la precarización del trabajo. Las fábricas de la charla en Chile son un claro ejemplo de las formas que asume la organización del trabajo en el escenario laboral contemporáneo.

Palabras clave: call centers, materialidad del trabajo, reproducción, subjetividad laboral.

Abstract: This article presents the preliminary results of PhD's research aimed at discovering the subjetivity and materiality of the work in the Call Centers focus in Chile. A salary defined with unattainable goals, abuse, and exhaustion of a new type of double extraction: productive and reproductive value as women's workers and their families. Also the exploitation of subjective capacities, among other things, show new forms that asssumes job's insecurity and precariousness. The talking factories as a clear example of the forms which take place by the organization of work in the contemporary labor conditions scene.

Keywords: call centers, labor reproduction, materiality of the work, work's subjetivity.

* Doctoranda en Investigación Social y Cultural. 
Este artículo expone los resultados preliminares de la investigación de tesis doctoral en Antropología social y cultural orientada a conocer la organización del trabajo y la subjetividad laboral de los trabajadores/as de los call centers subcontratistas en Chile.

En las últimas décadas, la crisis del fordismo taylorismo como modelo dominante de organización del trabajo y la instalación de formas diversas de postfordismo, han generado profundos cambios en las modos de producir y de organizar el trabajo a nivel mundial. Si bien se observan procesos diversos y heterogéneos en el mundo del trabajo contemporáneo, es posible mencionar algunas tendencias globales relevantes para este estudio. Por una parte, se evidencia una nueva división global del trabajo, que reorganiza la producción a nivel mundial fragmentando la producción en circuitos transnacionalizados, ${ }^{1}$ y por otra, se incorporan de manera creciente a los procesos productivos capacidades humanas subjetivas que no fueron centrales en los periodos anteriores de desarrollo capitalista (Antunes, 2002; Gounet, 1999; Virno, 2003). Se observa también la creciente disminución de la población activa ocupada en actividades productivas industriales ${ }^{2}$ y el aumento de la población ocupada en actividades de servicios, señalada por la OIT (Tendencias del trabajo mundial, 1999) como una de las principales tendencias mundiales del trabajo.

En este contexto global, la fragmentación productiva y la flexibilización de la producción y del trabajo, unida a la desregulación jurídica del trabajo y a una regresión en los derechos sociales y laborales, se asocian en gran parte del mundo occidental, especialmente en América Latina y particularmente en Chile, a una amplia precarización del trabajo (Antunes, 2002; De la Garza, 2000; Todaro; Yañez, 2004, entre otros).

En el escenario de las tendencias globales mencionadas y de la revolución de las tecnologías de la información (Castells, 1996), comienzan a instalarse los call centers o fábricas de la charla ${ }^{3}$ en la década de los 90',

1 En las últimas décadas el capital ha desconcentrado territorialmente el capital productivo logrando que las secciones nacionales se ocupen sólo de una parte del proceso productivo total. En la búsqueda de menores costos de producción, el capital se instala en países con escasas regulaciones en las condiciones ambientales y en la explotación de la fuerza de trabajo.

2 Si bien esta tendencia se observa a nivel mundial, es mayor en los países industrializados de Europa occidental, donde la ocupación en la industria bajó desde un 40\% en la década del 40 ’ a un 20-25\% a comienzos del año 2000 (Gorz, 1997).

3 En el análisis de los call centers en Argentina (Colectivo Situaciones, 2006) Paolo Virno denomina a los call centers fábricas de la charla.

Horizontes Antropológicos, Porto Alegre, ano 19, n. 39, p. 19-40, jan./jun. 2013 
entendidos como empresas basadas en plataformas telefónicas asociadas principalmente a empresas de servicios. ${ }^{4}$ A mediados de la década del 2000 los call centers empleaban a cerca de 8 millones de trabajadores/as en el mundo, concentrados fundamentalmente en EEUU -que representa cerca del 55\% de las fuentes laborales de los call centers a nivel mundial-, Inglaterra, Alemania y Francia. Como parte de los procesos de deslocalización, las empresas de call centers de estos países trasladan sus centros de llamados a países de América Latina, Asia y África, que cuentan con la infraestructura tecnológica necesaria y menores costos laborales (Kremerman, 2005). Así, en la década del 90' los call centers se instalan en diversos países de América Latina y el Caribe empleando a más de 330 mil trabajadores/as en el año 2005, especialmente en México, Brasil -que concentran más del $85 \%$ de la fuentes laborales de los call centers en Latinoamérica- y Argentina (Kremerman, 2005). También en países como India, Marruecos y crecientemente en Filipinas, ${ }^{5}$ se observa una importante actividad de estas fábricas de la charla.

A diferencia de Estados Unidos donde los call centers se encuentran en un periodo de desarrollo maduro y de países como México o Argentina donde se encuentran en etapa de crecimiento, en Chile estas empresas comienzan a instalarse a fines de la década del 90' y ya en el año 2005 se registraron entre 14 y 15 mil puestos de trabajo (Kremerman, 2005). Algunas grandes empresas en Chile incorporan call centers como parte de sus procesos productivos ${ }^{6} \mathrm{y}$ otras son empresas subcontratadas orientadas exclusivamente a la atención telefónica de otras empresas, fenómeno conocido como "outsourcing”. En algunos casos los call centers subcontratistas cuentan a su vez con trabajadores/as suministrados por otras empresas, en una extensa cadena de subcontratación.

Los call centers como empresas que forman parte de los circuitos de transnacionalización y deslocalización de la producción, que incorporan

4 "Un call center, principalmente se basa en la utilización de plataformas telefónicas y equipos conexos para mantener negocios y fortalecer la relación entre una empresa y sus clientes... consiste en una serie de operadores -humanos o automáticos- que reciben o emiten llamados telefónicos, apoyados en un software que permite realizar seguimiento de esas comunicaciones cuyo objetivo central puede ser la venta, la atención de reclamos, la cobranza, entre otros.” (Kremerman, 2005, p. 26).

5 Según los datos de Datamonitor report (2005), India concentra la mayor actividad de call centers en Asia pero Filipinas muestra un aumento creciente. Para el año 2009 se proyectaba que casi un cuarto de millón de nuevos puestos de agentes se incorporarían en ambos países.

6 Son aquellas empresas que necesitan e instalan un centro de llamados para vender productos, realizar encuestas, responder a las necesidades de sus clientes, entre otros objetivos.

Horizontes Antropológicos, Porto Alegre, ano 19, n. 39, p. 19-40, jan./jun. 2013 
fundamentalmente a mujeres jóvenes como trabajadoras e incluyen las capacidades subjetivas y de comunicación como eje central de su actividad, implican una profunda precarización del empleo y son un claro ejemplo de las nuevas formas que asume la organización del trabajo. Así, el habla puesto a trabajar en las fábricas de la charla -la identidad entre trabajo y comunicación verbal-, se posiciona como uno de los núcleos centrales del postfordismo en el mundo del trabajo actual (Virno, 2003).

Esta nueva realidad de los call centers en Chile me motivó a integrar el estudio de las condiciones materiales del trabajo, la subjetividad laboral y las relaciones sociales de género de estas empresas, en un análisis que pretende dar cuenta de la complejidad de la realidad laboral y social contemporánea. Por materialidad del trabajo entiendo las condiciones laborales de inserción de los trabajadores/as y los modos de producir y de organizar el trabajo de los call centers en el marco del capitalismo global, y por subjetividad laboral las formas de extrañamiento o enajenación, ${ }^{7}$ es decir, la relación de los trabajadores y trabajadoras de los call centers con la producción inmaterial de su trabajo, la relación de los sujetos con el trabajo y la relación entre los sujetos en el mundo laboral. La noción de extrañamiento que se utiliza en este estudio da cuenta también de la captura de las capacidades humanas subjetivas ${ }^{8}$ por el capital en las nuevas formas postfordistas de organización del trabajo.

Como procedimiento metodológico general utilicé, por una parte, un enfoque histórico que permitió comprender la instalación y desarrollo de los call centers en el marco de las dinámicas económico sociales de las últimas décadas en América Latina y Chile; y por otra, una perspectiva que vincula lo general y lo particular, enfatizando en la relación entre el contexto global de organización del trabajo en que se insertan las empresas estudiadas y la perspectiva de los/as propios/as sujetos/as respecto a su trabajo.

Desarrollé el trabajo etnográfico a través de la observación directa en las fábricas de la charla, la revisión de documentos y de entrevistas en profundidad realizadas a 40 trabajadores/as de 12 call centers de la región

\footnotetext{
Los conceptos de extrañamiento y enajenación son diferentes en términos filosóficos, sin embargo, para los fines de este estudio serán utilizados indistintamente. Estas nociones serán desarrolladas en el informe final de investigación.

8 Capacidades humanas subjetivas como la disponibilidad a prestar atención, a preguntar, a gestionar los afectos, la facultad de producir imágenes y relaciones, de producir organización y lectura de información y especialmente las capacidades de comunicación y uso del habla (Colectivo Situaciones, 2006).
}

Horizontes Antropológicos, Porto Alegre, ano 19, n. 39, p. 19-40, jan./jun. 2013 
metropolitana de Chile, tanto teleoperadores/as como supervisores/as y a dirigentas sindicales de la Federación Nacional de Trabajadoras de Call Centers FETRACALL.

\section{Una mirada inicial a las trayectorias laborales}

A pesar de ser en su mayoría personas jóvenes, ${ }^{9}$ casi la totalidad de los sujetos de investigación tienen una trayectoria laboral compleja, de largos años de trabajo en empleos parciales, vinculados en la mayor parte de los casos a trabajos del sector servicios en labores de administración, secretariado, ventas, promoción, cobro, entre otros. Las ocupaciones a las que acceden los sujetos entrevistados son trabajos precarizados, muchos de ellos sin contrato de trabajo, por tiempos parciales, con bajos salarios y extensas jornadas de trabajo. En el contexto general de precarización del trabajo, las condiciones laborales en los call centers resultan más atractivas para los trabajadores/as, como lo demuestra esta cita de una joven supervisora de Atento,

[...] me vieran como yo trabajaba antes, tenía aquí marcada la blusa, me vestía de señorita, andaba en la calle, pedirle al chofer que me llevara por 100 pesos, comía en una plaza. Caminar un montón para vender... Entonces yo llegué aquí, me entregaron una cartera de clientes, había aire acondicionado, me pagaban mi sueldo como correspondía. Cuando yo entré como operadora, el primer mes saqué cuatrocientas lucas, yo nunca en mi vida había visto tanta plata [...] (Marisela).

Es evidente que tanto la formación como la experiencia laboral anterior de los sujetos de investigación se vinculan estrechamente al tipo de actividades que se desarrollan en los call centers. Las habilidades necesarias para las diversas labores que se realizan en estos centros son similares a las aprendidas en los estudios técnicos y en las diversas actividades remuneradas de los trabajadores/as. Si bien los call centers se caracterizan por no exigir formación ni

\footnotetext{
9 La edad de los sujetos entrevistados, tanto teleoperadores/as como supervisores/as, fluctúa entre los 22 y los 35 años, con un promedio de 28 años. Excepcionalmente, en uno de los casos, la teleoperadora tiene más de 50 años de edad. Considerando que en Chile se reconoce formalmente como población joven a las personas entre 15 y 29 años de edad, es posible señalar que la fuerza de trabajo de los call centers está constituida por trabajadores/as jóvenes.
}

Horizontes Antropológicos, Porto Alegre, ano 19, n. 39, p. 19-40, jan./jun. 2013 
experiencia laboral previa, las habilidades requeridas para este tipo de trabajo forman parte de los aprendizajes insertos en los estudios y empleos masivos en esta etapa de ampliación de las actividades llamadas terciarias.

\section{Apuntes sobre condiciones laborales: contratos y salarios en los call centers ${ }^{10}$}

En el periodo de recopilación de la información, la mayoría de los sujetos entrevistados desarrolla sus labores de teleoperadores/as y supervisores/as con un contrato de trabajo indefinido. Sin embargo, en varios casos los trabajadores/as iniciaron sus trabajos en call centers con contratos a plazo fijo por tres o seis meses y posteriormente fueron traspasados a contratos indefinidos. Este formato, que se observa en las empresas suministradoras de trabajadoras/ es y en un call center, permite a las empresas definir el no traspaso a contrato indefinido según las evaluaciones del rendimiento de los teleoperadores/as. En estos casos los trabajadores/as son "desvinculados" de la empresa, figura que desdibuja la noción de "despido".

Los contratos de los trabajadores/as de call centers se modifican cuando las campañas de las empresas específicas a las cuales presta servicios el call center terminan o según las "necesidades de la empresa”. En estos casos habitualmente se establecen "anexos" o "adjuntos" a los contratos de trabajo, mediante los cuales se modifican las jornadas de trabajo - ampliándose o restringiéndose de 31 a 45 horas de trabajo de acuerdo a las necesidades de cada campaña- y especialmente las tablas de comisiones por ventas o los requerimientos necesarios para alcanzar el bono de calidad ${ }^{11}$ de la campaña específica, que constituye la parte variable del salario de los teleoperadores/as. Si bien algunos de los sujetos entrevistados señalan que los “anexos” al contrato sólo pueden establecer iguales o mejores condiciones de trabajo, en muchos casos las modificaciones de las condiciones para alcanzar el bono de calidad generan mayores exigencias en el cumplimiento de metas, disminución del tiempo

${ }^{10}$ Los antecedentes sobre la organización del trabajo en los call centers serán integrados en los resultados finales del estudio.

11 El bono de calidad constituye la parte variable del salario de los teleoperadores/as y determina las condiciones requeridas para su obtención. Habitualmente considera la asistencia, puntualidad, el Tiempo Medio de Operación TMO, la utilización de palabras no aceptadas, los errores en el procedimiento formal, entre otros. 
ocupado en la atención de cada llamada Tiempo Medio de Operación TMO, restricciones en las ausencias del trabajo, etc. Como lo refleja el testimonio de Mariana, teleoperadora de Atento, el aumento sistemático de las exigencias para obtener el bono de calidad, que constituye en algunos casos la mitad del salario de los teleoperadores/as, se sitúa progresivamente en límites difícilmente alcanzables por los trabajadores/as, lo que provoca la paulatina disminución de los salarios, que se restringen cada vez más a la parte fija del salario,

[...] el TMO es el tiempo que uno demora con el cliente en línea y ellos ponen un tiempo, que tiene que ser... generalmente ponen minuto y medio, minuto... sí más menos un minuto... un minuto 70 segundos, una cosa así. Porque todo es plata para ellos, entonces entre más se demore uno con el cliente más plata pierden ellos, entonces el TMO es para fijar un tiempo, si te pasas del tiempo no te ganas el bono; si tú llegas atrasado no te ganas el bono; si tú faltas o tienes muchas licencias en el mes no te ganas el bono. Los que estaban en ventas era comisión por ventas, nosotros en atención al cliente si teníamos bajo el TMO, bajo el tiempo que ellos decían, no nos ganábamos el bono [...] (Mariana, teleoperadora Atento).

Si bien algunos de los entrevistados señalan el logro de ciertos niveles de negociación en las condiciones establecidas en los "anexos" de los contratos, en otros casos -como menciona Marina, teleoperadora de Sermec-, existen presiones y amenazas de despido para lograr la aceptación de los cambios en las condiciones contractuales.

[...] nos hacen firmar un documento donde aceptamos la baja de la tabla de comisiones y si no aceptas te despiden. Despidieron a unos compañeros el año pasado, por necesidades de la empresa, dijeron que no les convenían porque tenían más de un año, pero todos sabemos que fue porque no quisieron firmar la baja de comisiones [...]

Al parecer, la experiencia laboral anterior, el manejo de los procedimientos de los call centers, la participación en sindicatos, entre otros factores, junto a la flexibilidad empresarial en el establecimiento de contratos de trabajo "individualizados", generan ciertos niveles de negociación diferenciados con cada trabajador/a.

Los salarios de los teleoperadores/as de call centers se componen de una parte fija y una parte variable: la parte fija del salario está estipulada en el

Horizontes Antropológicos, Porto Alegre, ano 19, n. 39, p. 19-40, jan./jun. 2013 
contrato de trabajo y definida por el sueldo mínimo que cada año se establece en la negociación entre sindicatos, empresarios y el Estado; y la parte variable del salario se menciona en el "anexo" del contrato de trabajo e indica, según la campaña específica en que labora cada teleoperador/a, la tabla de comisiones por venta o los requerimientos para acceder al bono de calidad.

Los teleoperadores/as con mayor experiencia laboral en el sector y las dirigentes sindicales de la FETRACALL señalan que los salarios han disminuido progresiva y sistemáticamente a partir de la utilización del TMO a principios del 2000 y asimismo con el progresivo aumento en las exigencias para la obtención del bono de calidad como lo señala Francisca, teleoperadora de Sermec.

[...] al principio igual se ganaban buenas lucas [pesos chilenos], daban ganas de venir no faltar porque daban bonos por no faltar ni un día, daban otros bonos por nota y después empezaron a bajar los bonos, si antes ganabas 350 mil pesos y ahora ganas con suerte $250 .$. y más o menos esa es la relación... ellos te ofrecieron tanta plata y después fueron cambiando las reglas del juego [...]

El aumento progresivo de los requerimientos para acceder al bono de calidad -que lo sitúa en lugares cada vez más inalcanzables- y los masivos errores en los cálculos salariales de los teleoperadores/as, generan incertidumbre e inseguridad en la mayoría de los entrevistados/as e implican la sistemática disminución de los salarios de los teleoperadores/as, el aumento de la productividad y de las ganancias del capital, y la emergencia de nuevas formas de explotación de la fuerza de trabajo.

Si bien los salarios de los teleoperadores/as han disminuido sostenidamente en los últimos años, la mayoría de los sujetos entrevistados coincide en señalar que los salarios de los call centers son comparativamente más altos que los obtenidos en otros ámbitos laborales. Los salarios más bajos obtenidos por Isabel y Claudia en diversos trabajos del sector servicios, dan cuenta de esta realidad,

[...] lo que pasa es que yo estudié turismo, trabajé en agencias de viajes también en venta de intangibles y de vendedora, pero en cuanto al sueldo, yo primera vez que ganó máximo 300 [...] (Isabel, teleoperadora de Sermec).

[...] en el fondo tú ves el mercado y más que eso una no gana, las pegas están entre 350 y 400 lucas máximo, yo estuve trabajando siete años en un supermercado

Horizontes Antropológicos, Porto Alegre, ano 19, n. 39, p. 19-40, jan./jun. 2013 
y lo más que puede ganar son 180, igual te mata lo de los supermercados y tienes que trabajar los domingos. (Claudia, teleoperadora Multivoice).

Efectivamente, los salarios de los trabajadores/as de call centers son superiores al salario mínimo en Chile, inclusive en el caso de más baja remuneración que corresponde a una jornada parcial de 18 horas semanales. Sin embargo, la dinámica salarial de los call centers forma parte de las tendencias generales del país que indican una distribución funcional del salario que se invierte desde la década del 70' -cuando el 63\% de las ganancias iba al trabajo y el 37\% al capital- hasta la década del 2000 cuando sólo el $40 \%$ de las ganancias va al trabajo y el $60 \%$ al capital. ${ }^{12}$ Es decir, si bien los salarios de los trabajadores/as de las fábricas de la charla son comparativamente más altos que en otros empleos precarizados del sector servicios, siguen formando parte de una dinámica general de explotación de la fuerza de trabajo y de aumento de las ganancias del capital.

Según los datos obtenidos, y en concordancia con lo señalado por los sujetos entrevistados y las dirigentes sindicales del sector, no se observan diferencias entre los salarios de hombres y de mujeres, tanto en el caso de los teleoperadores/as como de los supervisores/as.

\section{Inestabilidad e incertidumbre en las fábricas de la charla}

Una de las características principales de los call centers estudiados en otros países del capitalismo periférico es la alta rotación de los teleoperadores/ as. La gran mayoría de los sujetos entrevistados reconoce esta dimensión y asevera que los ejecutivos no laboran habitualmente más de seis meses en un mismo call center. La rotación masiva de los teleoperadores/as se generaría por la no renovación de contrato ante el incumplimiento de las requerimientos de la organización del trabajo -bajas evaluaciones en los bonos de calidad, incumplimiento en las metas de ventas, etc.-, y también por la deserción de los propios teleoperadores/as ante la insatisfacción por las jornadas de trabajo o los bajos salarios generados por la imposibilidad de cumplir las metas impuestas.

12 En entrevista a Hugo Fazio citada en Escobar Salazar y Kries (2005). 
[...] hay mucha rotación, mucha, mucha gente se va o la echan. Han echado a harta gente por disponibilidad, puntualidad, el tiempo que deberían estar conectados, que sacan la vuelta, y por calidad -a nosotros nos hacen creo que son como 8 o 7 grabaciones en escucha y ponen nota, si tienes malas notas durante tres meses te pueden despedir. En este tiempo han echado a hartas personas por esa razón [...] (Marina, teleoperadora Sermec).

Los supervisores/as entrevistados en este estudio tienen una antigüedad laboral entre los 4 y 9 años en las empresas que trabajan actualmente, sin embargo, algunos trabajadores/as, como Mariana teleoperadora de Atento, señalan que la rotación también ocurre en este nivel de trabajadores/as de call centers, al parecer de manera menos masiva.

[...] me asignaron un supervisor, no habían pasado ni cuatro meses y nos cambiaron el supervisor entonces uno no alcanzaba a conocer a su supervisor, a tenerle confianza cuando ya ipum! le cambiaban supervisor, en menos de un año tuve como cinco supervisores $[\ldots]$

La rotación de los trabajadores/as se explica también por los despidos masivos que implica la dinámica de deslocalización de los call centers. Las empresas que requieren los servicios de call centers movilizan sus campañas y capitales hacia los países que les aseguren menores costos y mayor acumulación de ganancias, lo que se observa -como señalan las dirigentes sindicales de FETRACALL-, en el movimiento de campañas desde call centers instalados en Chile hacia empresas ubicadas en otros países de la región, especialmente en Perú y Ecuador.

Si bien los antecedentes de los call centers y la percepción de los sujetos entrevistados/as coinciden en señalar una amplia y masiva rotación de trabajadores/as en estas empresas, en los casos estudiados no se observa esta tendencia, con un promedio de tres años en el trabajo actual en el caso de los teleoperadores/as y de cuatro años en el caso de los supervisores/as. De los cuarenta sujetos entrevistados sólo la mitad cuenta con experiencia laboral en call centers, en la mayoría de los casos en una empresa. Sólo en algunos pocos casos los teleoperadores/as trabajaron en tres o cuatro empresas de call centers con anterioridad a sus empleos actuales, en un movimiento de búsqueda de mejores condiciones laborales. No se dispone en Chile de datos concretos que permitan conocer las tendencias de las fábricas del habla en las últimas 
décadas, sin embargo, es posible sostener inicialmente que la tendencia que durante los primeros años identificaba a los call centers en el país como nichos de trabajo parcial e inestable y de ocupación fundamentalmente de estudiantes, se transforma progresivamente. Se observa una nueva y compleja dinámica que reúne por una parte a trabajadores/as de call centers que son incorporados y expulsados del mercado de trabajo, y por otra, a trabajadores/as que al "adaptarse” a los procedimientos y requerimientos de las fábrica del habla, logran mantener una antigüedad laboral y salarios más altos que en otros empleos del sector servicios. Desde la perspectiva de las dirigentes sindicales de FETRACALL, el perfil del teleoperador/a de call centers se modificó en los últimos años del estudiante que buscaba un empleo parcial y de bajo salario, a mujeres -habitualmente jefas de hogar-, que requieren estabilidad laboral y salarios para mantener la economía de su unidad doméstica.

[...] se van de una empresa a otra porque los echan... o se aburren con los horarios de noche o la mala onda con los supervisores, como son tan rotativos hay estudiantes que trabajan uno o dos meses en cada call center... pero ahora se quedan varios años. Nosotros tenemos trabajadoras de 4, 5 años, nosotras mismas ya cumplimos los tres. Yo creo que después de la negociación colectiva el trabajo se volvió más estable porque antes se veía más deserción [...] (dirigenta sindical Multivoice).

Los datos disponibles de la muestra del estudio avalan esta tendencia: sólo tres sujetos son estudiantes y la gran mayoría mantiene el trabajo en los call centers como su actividad asalariada principal.

\section{Nuevas formas de precariedad laboral: maltrato y "cansancio de nuevo tipo" en los call centers}

La mayoría de los teleoperadores/as, especialmente los que se ocupan en plataformas de atención al cliente -segunda atención a los clientes en caso de reclamo por un servicio-, señalan sufrir maltrato en el trabajo por parte de los clientes tanto de campañas que prestan servicios en Chile como en otros países. Al parecer, los procesos de deslocalización de las fábricas del habla -que sitúan a trabajadores/as en la atención de servicios en otras localidades y culturas- generan tensiones en las relaciones entre los teleoperadores/as y 
los clientes de las empresas contratantes de los servicios de call centers. Como ejemplifica el testimonio de Javier, teleoperador de Teleperformance, los trabajadores/as entrevistados mencionan discriminación por parte de los clientes no nacionales.

[...] en atención al cliente donde estoy yo, nos dicen "sudaca no quiero nada con ustedes, son inoperantes...” inmediatamente un choque frontal con el cliente, por ser nosotros extranjeros, el cliente español nos trata muy mal, depende del estado de ánimo, yo regularmente no estoi’ ni ahí, no pesco ahora [no le da importancia].

Considerando que el maltrato ocurre generalmente por el reclamo en la calidad de los servicios de las empresas, es posible sostener que los trabajadores/as asumen frente a los clientes la identidad empresarial, sufren en la vida laboral cotidiana el impacto de la agresividad generada por la disconformidad de los clientes y enfrentan con recursos y habilidades personales el maltrato. Los sujetos entrevistados sostienen que logran controlar estas situaciones, sin embargo, su ocurrencia en la vida laboral cotidiana es uno de los factores que incide en el cansancio de nuevo tipo que se evidencia en los trabajadores/as del sector.

En muchos casos las relaciones laborales entre teleoperadores/as y supervisores/as se caracterizan también por el abuso de autoridad y el maltrato. Estas condiciones del ambiente laboral se sitúan como parte de las malas condiciones laborales de los trabajadores/as de call centers y de las nuevas dimensiones y formatos que asume la precariedad laboral actual.

La mayoría de los sujetos entrevistados -tanto teleoperadores/as como supervisores/as- señala que el trabajo en los call centers no genera problemas relevantes de salud física. Sin embargo, la mayoría de ellos señala el cansancio, los trastornos del sueño y el stress como los principales problemas de salud laboral. La intensidad del trabajo -especialmente en las plataformas de entrada de llamados-, el escaso descanso durante la jornada laboral, la tensión permanente que genera el conflicto con los usuarios, la dinámica repetitiva del trabajo, la presión de los supervisores/as, la urgencia de resultados para obtener el salario esperado, y en algunos casos la sobrecarga de trabajo y estudios, son mencionados como los principales factores que generan stress, cansancio y agotamiento mental en los teleoperadores/as de call centers. 
[...] físicamente es lo más liviano que hay. Pero el tiempo que estuve en la parte comercial, mentalmente estaba agotado. Estresa bastante. En algunos momentos me sentía bastante cansado. Y la mayoría tenía la misma sensación. Quedaba cansado, quedaba agotado. Lidiar con los conflictos de otras personas o escuchar algo por obligación que no quieres escuchar, eso es lo que te agota [...] (Juan, teleoperador Emergia).

La doble jornada de trabajo productivo y reproductivo ${ }^{13}$ de la mayoría de las mujeres entrevistadas es también un factor que incide en el cansancio de las trabajadoras. Si bien las trabajadoras cuentan con un soporte familiar importante en el desarrollo del trabajo doméstico y de cuidado de los hijos/as, le agrega un factor más de sobrecarga a la fuerza de trabajo femenina. A pesar de que algunas investigaciones sostienen que los sectores juveniles en Chile son más proclives a realizar cambios en los roles socialmente asignados a hombres y mujeres (Gutiérrez; Osorio, 2008; Instituto Nacional de la Juventud, 2010), en el caso de los trabajadores/as de call centers que forman parte de una fuerza de trabajo juvenil, no se observa esta tendencia, posiblemente por su convivencia con las familias nucleares en la mayoría de los casos.

Casi todas las personas entrevistadas señalan el cansancio y el agotamiento como característica del trabajo de las fábricas de la charla, pero mencionan también periodos de mayor o menor intensidad del trabajo y la posibilidad de moverse al interior del call center en diferentes plataformas que les permitan una dinámica laboral menos estresante.

En los periodos de mayor intensidad del trabajo, el cansancio y el stress laboral tienen un impacto negativo en la vida doméstica y familiar. La irritabilidad, el desánimo, la pérdida de paciencia, entre otros, afectan la vida personal de los/as ejecutivos/as y las relaciones con sus hijos/as.

Ese agotamiento que los sujetos mencionan parece ser un "cansancio de nuevo tipo" que se vincula a la utilización intensiva de las capacidades humanas subjetivas. A diferencia del fordismo clásico, en que el agotamiento obedecía más bien a un cansancio físico generado por la explotación de las capacidades y habilidades manuales, en las fábricas de la charla se explotan capacidades humanas vinculadas a la comunicación y a las capacidades emocionales. Las necesidades de prestar atención, comprender problemas,

13 Trabajo asalariado en los call centers y trabajo doméstico en su unidad doméstica.

Horizontes Antropológicos, Porto Alegre, ano 19, n. 39, p. 19-40, jan./jun. 2013 
resolver conflictos, mediar, seducir, negociar soluciones, convencer, contener, realizar tareas múltiples, etc., bajo un control rígido del tiempo, genera en los trabajadores/as de call centers un cansancio de más bien de tipo mental y emocional, que afecta también su vida doméstica.

\section{Unidad doméstica y trabajo reproductivo}

Considerando que la fuerza de trabajo de los call centers es fundamentalmente población joven y que habitualmente los/as jóvenes se independizan tardíamente de su familia en Chile, es comprensible que la gran mayoría de los sujetos de la investigación constituya su unidad doméstica en convivencia con su familia de origen, habitualmente las madres, padres, hermanos/as y abuelos/as. La convivencia con la familia de origen es un soporte relevante para la vida cotidiana de los trabajadores/as en cuanto permite compartir el trabajo doméstico, la crianza de los hijos/as, y también los costos de la economía doméstica.

En casi todos los casos las mujeres asumen la crianza de los hijos/as con el apoyo de sus madres, abuelas, y en algunos casos, de hermanos/as mayores. Sólo en algunos casos de trabajadoras con una relación de pareja estable se observa la participación de los hombres en el trabajo doméstico y de cuidado de los hijos/as. En los casos de trabajadoras con pareja estable o sin ella se evidencia que los padres de los hijos/as de las trabajadoras tienen un rol mínimo o nulo tanto en el mantenimiento económico como en el cuidado y crianza de los niños/as.

La mayoría de las trabajadoras asume las tareas de trabajo doméstico y de crianza de los hijos/as como parte de sus roles centrales, sin embargo asumen una actitud crítica frente a la escasa o nula responsabilidad de los hombres en estos trabajos.

El cumplimiento de los derechos maternales que se constata en las fábricas del habla a través de la financiación de salas cunas o cuidadoras para los/as menores, permite también un soporte en el cuidado de los hijos/as. A diferencia de muchas empresas en Chile que utilizan estrategias para evitar cumplir los derechos maternales, todas las trabajadoras entrevistadas señalan que en los call centers se respetan los periodos de pre y posnatal y se financian los costos de salas cunas o cuidadoras para sus hijos/as. 
Se observa entonces una co-responsabilidad en el trabajo y economía doméstica y también en la crianza de los hijos/as, lo que en estricto rigor no sitúa a las mujeres entrevistadas como “jefas de hogar”, sino más bien como partes de una densa red de colaboración familiar que se instala como un soporte importante ante los salarios variables de los trabajadores/as de call centers.

Uno de los atractivos mencionados por las trabajadoras entrevistadas para buscar empleo y mantenerse en las empresas de call centers es la posibilidad de conciliar el trabajo doméstico con el trabajo remunerado. Las jornadas de trabajo parciales, la posibilidad de modificar el contrato laboral y disminuir la jornada de 45 horas semanales, la flexibilidad horaria en algunas empresas, son destacadas como factores fundamentales para conciliar el trabajo asalariado con el cuidado y crianza de los hijos/as.

[...] yo creo que es igual para la mayoría de las mujeres... yo por lo menos busco este trabajo porque uno no quiere dejar de lado la familia tampoco y trata de aportar en algo para la casa, si hubiera sido de otra forma busco un trabajo de lunes a viernes con un horario más largo, pero al final tampoco es tanta la plata que uno gana. (Edith, teleoperadora Sermec).

La constatación de que la gran mayoría de las trabajadoras entrevistadas realiza el trabajo asalariado en los call centers junto al trabajo doméstico y de crianza de los hijos/as, permitiría sostener que se observa en las fábricas de la charla una doble extracción del valor del trabajo productivo y reproductivo de las mujeres trabajadoras. Sin embargo, la participación central de la familia en el trabajo reproductivo incorpora una nueva dimensión a la explotación de la fuerza de trabajo. En estos casos, el capital no sólo utiliza el trabajo reproductivo de las trabajadoras de los call centers, sino también el de otras mujeres ${ }^{14}$ que componen la unidad doméstica. La explotación de la fuerza de trabajo se extiende por lo tanto a la unidad doméstica a través de los roles de género socialmente asignados a las mujeres en las sociedades occidentales.

\footnotetext{
14 Si bien en algunos casos, se menciona el trabajo de abuelos y hermanos mayores de las ejecutivas de call centers en el trabajo doméstico y de cuidado y crianza de los hijos/as, habitualmente lo realizan las madres y abuelas de éstas.
} 


\section{Habilidades y capacidades humanas explotadas en las fábricas de la charla}

Las diferentes funciones que cumplen los teleoperadores/as y los supervisores/as en las fábricas de la charla establecen diferencias en las habilidades y capacidades necesarias para sus respectivos trabajos. Se observan habilidades comunes en el caso de los teleoperadores/as, pero también énfasis diversos dependiendo de las plataformas y tareas específicas que estos/as deben desarrollar.

En los trabajos vinculados a la atención directa de los clientes, se exige de los teleoperadores/as la capacidad de comprender las necesidades del usuario de los servicios y de resolver los problemas identificados en el menor tiempo posible. Como se evidencia en la entrevista a Juan, teleoperador de Emergia, la capacidad de interpretar los requerimientos, identificar y dar rápida solución a los problemas planteados, requiere la atención permanente y agilidad mental de los trabajadores/as de estas plataformas.

[...] si es atención con personas en el momento, necesitas comprensión o hacer una síntesis de lo que necesitan. Porque si uno habla con una persona, algunos van directo al grano a lo que necesitan, son directos. Con otras personas uno puede estar hablando media hora y no sabes que es lo que necesitan. Y tienes que ser capaz de sintetizar lo que te está pidiendo y darle una respuesta [...]

En las plataformas de recepción y atención de reclamos, los ejecutivos/as sufren constantemente agresiones y malos tratos. En estos casos los trabajadores/as desarrollan las capacidades de contención de la agresividad, de mediación entre los requerimientos de los usuarios y las normativas empresariales y de negociación en la resolución de conflictos.

En las plataformas orientadas a las ventas y a la contención ${ }^{15}$ de los clientes en las empresas se utilizan fundamentalmente las capacidades de convencimiento y de seducción. Por una parte los teleoperadores/as utilizan su propia experiencia como consumidores/as para enfatizar las características de los productos que los transforman en algo “deseable”. La búsqueda de argumentos creíbles y la propia convicción sobre la calidad del producto, se mencionan

${ }^{15}$ La organización de los call centers habitualmente cuenta con varias plataformas o espacios de trabajo diferenciados por funciones específicas. En la mayoría de los call centers se observan plataformas de ventas, cobranzas, de contención (evitar que los usuarios terminen los contratos de servicios), de atención al cliente o reclamo, entre otros.

Horizontes Antropológicos, Porto Alegre, ano 19, n. 39, p. 19-40, jan./jun. 2013 
en estos casos como habilidades básicas para el logro de buenos resultados. Por otra parte, los teleoperadores/as intentan establecer una relación de cercanía personal con los clientes que les facilite el logro de las ventas. La cordialidad, la simpatía, e incluso la coquetería, son mencionadas como estrategias habituales para establecer en corto tiempo una relación distendida con los clientes. Como señala Edith, teleoperada de Sermec,

[...] tenemos que generar la instancia de tratar de hacer la conversación agradable con el cliente, porque toda negociación es fría, entonces uno obviamente tiene que romper esos esquemas con quién está hablando, ser simpática, dulce.

En estos casos, descubrir los dispositivos que gatillan el deseo de consumo augura resultados positivos para trabajadores/as que se desenvuelven personal y laboralmente en el contexto de una sociedad de masificación del consumo que opera con la lógica del deseo. La empatía y la capacidad de contención emocional con los clientes son mencionadas en algunos casos como habilidades centrales especialmente para el trabajo en campañas de asistencia.

A nivel general, los trabajadores/as requieren ciertas habilidades transversales, comunes a todos los teleoperadores/as de call centers. Las capacidades de comunicación, el manejo a nivel de usuario de las plataformas computacionales, y especialmente la "sonrisa telefónica" -como símbolo de la obligatoria atención amable y cordial con los usuarios-, son mencionadas por la gran mayoría de los sujetos entrevistados como habilidades básicas para desempeñarse en las fábricas de la charla.

Necesitas cosas super básicas: hablar bien, manejar el computador, ser constante... No es un trabajo muy difícil ipara nada, para nada! Un niño chico de 15 años podría trabajar perfectamente en un call center, sin ningún problema. (Mario, teleoperador Spa).

Muchas de las habilidades requeridas por los teleoperadores/as de los call centers -tales como la capacidad de convencimiento, la seducción, la mediación, la empatía, entre otras-, son reconocidas socialmente como características asociadas al género femenino en las sociedades occidentales de las cuales forma parte Chile. La utilización masiva de fuerza de trabajo femenina en los call centers -en Chile así como en la mayoría de los países con presencia de estas empresas- podría entonces vincularse con la explotación de las habilidades reconocidas socialmente como "propiamente femeninas". 
Además, las capacidades requeridas para el trabajo de los teleoperadores/as en los call centers, tanto a nivel transversal como en las plataformas específicas, son, en la mayoría de los casos, habilidades humanas básicas, que no requieren una calificación especializada. Por una parte, las capacidades de comunicación, de comprensión, de resolución de problemas, de síntesis, de convencimiento, etc., son habilidades habitualmente utilizadas en los empleos vinculados al sector servicios. La particularidad de las fábricas de la charla pareciera estar en la explotación intensiva de estas capacidades cognitivas y en la comunicación a distancia, que le otorgan una dinámica diferente de otros empleos del sector servicios. Por otra parte, en una sociedad de gran impacto de las tecnologías de la información y de considerable alfabetización digital como es Chile, el conocimiento computacional básico es de amplio manejo, especialmente en la población más joven del país.

El requerimiento de habilidades humanas básicas que no implican especialización específica pero si de habilidades socialmente reconocidas como “femeninas”, podría explicar en parte el ingreso masivo de fuerza de trabajo femenina, juvenil y con escasa capacitación a los call centers en Chile.

Los supervisores/as de los call centers, como trabajadores/as responsables de un grupo de teleoperadores/as de una plataforma ${ }^{16}$ y campaña específica, requieren habilidades diferentes a los ejecutivos/as. La mayoría de ellos/ as menciona la capacidad de liderazgo, la motivación en el manejo del grupo y el relacionamiento personal y afectivo con cada uno de los teleoperadores/as a su cargo, como las habilidades centrales utilizadas en su trabajo.

Todos los supervisores/as entrevistados coinciden en señalar la capacidad de liderazgo como la principal habilidad necesaria para desempeñar su labor. Un liderazgo que controla pero que no asume un perfil autoritario, que genera confianza y motivación grupal para el logro de las metas es, según los sujetos entrevistados, el tipo de liderazgo necesario para el manejo de grupos en las fábricas de la charla.

Otra característica central en el trabajo de los supervisores/as es la relación personal y afectiva establecida con cada uno de los ejecutivos/as a su

${ }^{16}$ La organización de los call centers habitualmente cuenta con varias plataformas o espacios de trabajo diferenciados por funciones específicas. En la mayoría de los call centers se observan plataformas de ventas, cobranzas, de contención (evitar que los usuarios terminen los contratos de servicios), de atención al cliente o reclamo, entre otros.

Horizontes Antropológicos, Porto Alegre, ano 19, n. 39, p. 19-40, jan./jun. 2013 
cargo. La lógica de flexibilidad que se impone en la dinámica de trabajo en cuanto a horarios, organización de las jornadas, evaluación, entre otros, otorga a los supervisores/as amplios ámbitos de decisión y poder. Para el manejo de estas normas, los supervisores/as utilizan el conocimiento de la vida personal de los trabajadores/as a su cargo y establecen una relación de carácter personal y afectivo. Como señala Marisela, supervisora de Atento,

un supervisor no cumple solamente la labor de administrador, hay que ser duro con las metas y blando con las personas y eso requiere la habilidad de trabajar sicológicamente con una persona, uno termina convirtiéndose en padre, en maestro, párroco, sicólogo, médico... Porque se trabaja con una persona que depende de uno para muchos factores, para retirarse antes, para poder estudiar... entonces uno necesita conocerlos muy bien, saber qué les pasa cada día.

Todos los supervisores/as entrevistados/as coinciden en señalar el conocimiento de la vida privada y la cercania personal con los trabajadores/as a su cargo como un ámbito fundamental de su trabajo que se utiliza más bien como una iniciativa personal que como orientación o normativa empresarial. En este sentido, los sujetos señalan que el aprendizaje de este procedimiento no se genera a través de los canales formales de capacitación institucional sino como parte de la misma experiencia laboral en el sector.

Si se evidencia una alta rotación de teleoperadores/as en las fábricas de la charla es posible preguntarse cómo logran establecerse las relaciones personales necesarias para organizar el trabajo de los supervisores/as. Más aún, algunos supervisores/as incluso mencionan estas relaciones personales y afectivas como mecanismo para impedir la rotación de los ejecutivos/as. Es posible que la tesis de la progresiva disminución de la rotación laboral y el aumento de trabajadores/as relativamente estables en los call centers, permita comprender esta paradoja.

\section{Identificaciones en las empresas de call centers}

Si bien casi la totalidad de los entrevistados/as -tanto teleoperadores/ as como supervisores/as- se identifica de manera espontánea con los perfiles que señalan sus contratos de trabajo -ejecutivos telefónicos, teleoperadores, operadores, entre otros- se reconocen a sí mismos como trabajadores. El 
cumplimiento de un horario de trabajo, el cansancio que genera, el funcionamiento jerárquico de la empresa, son señalados por los sujetos entrevistados/ as como factores que los identifica claramente como trabajadores/as.

La amplia y diversa trayectoria laboral de la mayoría de los sujetos entrevistados no genera la identificación con un oficio o ámbito laboral específico sino con la identidad general de "trabajadores/as". Si bien se reconocen como individuos que viven del trabajo e identifican las condiciones de precariedad del trabajo en los call centers, no se evidencia una identificación común como seres sociales que viven del trabajo. Se otorga mayor relevancia a un perfil individual vinculado con los procesos generales de individualización que se observan a nivel societal.

El trabajo en las fábricas de la charla se caracteriza por formar parte de una red de subcontratación que genera la vinculación directa de los trabajadores/as por lo menos con dos empresas diferentes: la empresa de call centers que contrata a los trabajadores/as y organiza el trabajo y la empresa para la cual prestan sus servicios. Estas complejas cadenas de subcontratación son conocidas por la gran mayoría de los trabajadores/as entrevistados. Al parecer, las dinámicas de subcontratación extendidas hace décadas en el mundo laboral chileno y las amplias trayectorias laborales de los entrevistados, les permite reconocer estas dinámicas del trabajo. A pesar de la vinculación directa con diversas empresas en el mundo de las fábricas de la charla, la mayoría de los trabajadores/as entrevistados se identifica con la empresa de call center y no con la empresa para la cual prestan servicios.

La mayoría de los teleoperadores/as entrevistados señala no sentirse parte de la empresa de call center en que labora. La precariedad de las condiciones laborales y especialmente la inestabilidad e inseguridad laboral que constatan en las fábricas del habla son mencionadas como los principales factores que generan una identificación más cercana a los trabajadores/as que con la empresa.

A diferencia de los teleoperadores/as, entre los supervisores/as se observa una identificación más bien ambigua con la empresa. Si bien la mayoría de los supervisores/as se reconoce a sí mismo como trabajador/a, se evidencian posicionamientos diversos en torno a la empresa: algunos de ellos señalan sentirse parte importante de la empresa, otros se sitúan en un espacio de intermediación entre la empresa y los teleoperadores/as, y otros aseveran no sentirse identificados con la empresa por las precarias condiciones laborales.

Horizontes Antropológicos, Porto Alegre, ano 19, n. 39, p. 19-40, jan./jun. 2013 
La mayoría de los entrevistados/as no se siente parte de la empresa en la que trabajan. Si bien no se observa una identificación ni individual ni colectiva con los objetivos empresariales, tampoco se evidencia una clara noción de las contradicciones entre capital y trabajo.

A diferencia de otros sectores laborales en Chile en los que se evidencian estrategias empresariales orientadas a lograr la identificación de los trabajadores/as con la empresa (Escobar Salazar, 2004), en los call centers no se observa la implementación de políticas o iniciativas en esta dirección. Al parecer, la lógica de aumento de las ganancias del capital no opera a través de la mayor explotación de una fuerza de trabajo "comprometida” con los objetivos del capital sino a través de otros mecanismos, como la explotación intensiva de las capacidades humanas subjetivas y la utilización de las habilidades reconocidas como "femeninas".

Estos resultados preliminares sobre los call centers en Chile permiten evidenciar nuevas formas de precarización del trabajo en el sector servicio, la explotación del trabajo productivo y reproductivo de no sólo de las trabajadoras sino también de las mujeres de su red familiar, y la utilización de las capacidades humanas más básicas y de los afectos que se instalan como nuevos mecanismos de explotación de la fuerza de trabajo y de aumento de las ganancias del capital.

\section{Referencias}

ANTUNES, R. Os sentidos do trabalho: ensaio sobre a afirmaçâo e a negaçâo do trabalho. Sao Paulo: Boitempo, 2002.

CASTELLS, M. La era de la información: vol. 1: la sociedad red. Madrid: Alianza Editorial, 1996.

COLECTIVO SITUACIONES. ¿Quién habla?: lucha contra la esclavitud del alma en los call centers. Buenos Aires: Ediciones Tinta Limón, 2006.

DATAMONITOR REPORT. The future of contact center outsourcing in India and the Philippines. 2006. Disponible en: <http://www.datamonitor.com/ technology>. Acceso: 26 oct. 2009.

DE LA GARZA, E. (Comp.). Reestructuración productiva, mercado de trabajo y sindicatos en América Latina. Buenos Aires: CLACSO, 2000. 
ESCOBAR SALAZAR, A. Trabajo, desocupación y configuración de la subjetividad laboral en la región del Bío-Bío-Chile. Tesis (Master)-Facultad de Ciencias Sociales, Universidad de Concepción, Concepción, 2004.

ESCOBAR SALAZAR, A.; KRIES, S. Desocupación en Chile en el marco de la mundialización 1990-2002: su carácter e impacto en la subjetividad. Concepción: Colectivo de mujeres América, 2005.

GORZ, A. Miserias del presente, riqueza de lo posible. Madrid: Paidós, 1997.

GOUNET, T. Fordismo e toyotismo na civilização do automóvel. São Paulo: Boitempo, 1999.

GUTIÉRREZ, E.; OSORIO, P. Modernización y transformaciones de las familias como procesos del condicionamiento social de dos generaciones. Revista Última Década, Santiago de Chile, n. 29, p. 103-135, 2008.

INSTITUTO NACIONAL DE LA JUVENTUD. Sexta encuesta nacional de juventud. Santiago de Chile, 2010.

KREMERMAN, M. En la ruta del trabajo decente: análisis de los sectores salmonicultura, call center y agroexportación. Santiago de Chile: Terram publicaciones, 2005. (Registro de problemas públicos, Informe n. 20).

TENDENCIAS DEL TRABAJO MUNDIAL. Revista OIT, Santiago de Chile, n. 31, sept./oct. 1999.

TODARO, R.; YAÑEZ, S. (Ed.). El trabajo se transforma: relaciones de producción y relaciones de género. Santiago de Chile: Centro de Estudios de la Mujer, 2004.

VIRNO, P. Virtuosismo y revolución, la acción política en la época del desencanto. Madrid: Traficantes de Sueños, 2003. 